\title{
Optimal combination of integral solutions of gradiometric boundary value problem using variance component estimation in Earth gravitational modelling
}

\author{
Mehdi Eshagh \\ Division of Geodesy, Royal Institute of Technology, SE 10044 Stockholm, Sweden \\ (Received February 16, 2009; Revised June 1, 2009; Accepted January 19, 2010; Online published July 9, 2010)
}

\begin{abstract}
Optimal estimation of geopotential coefficients is an important aspect of gravitational field recovery using satellite gravity gradiometry. The combination of gradiometric data and the use of tensor spherical harmonics is useful in this field. Here, we present a new strategy for combining different spectral solutions of the gradiometric boundary value problem by defining and formulating degree-order variance components and using the condition adjustment model. Numerical results show that the spectral combination of considering one degree-order variance component for each type of observation yields better results than the case where one degree-order variance component is estimated for each integral solution of the gradiometric boundary value problem. In this study, the estimates of the variance components are not considered in the standard way; rather, these components are mainly used to absorb the discretization error of the integral solutions. This method is capable of combining integrals in geosciences disciplines.
\end{abstract}

Key words: Degree-order variance component, optimal solution, degree of resolution, re-weighting process, stochastic model.

\section{Introduction}

The Earth's gravitational or magnetic potential can be approximated by a truncated series of spherical harmonics. The spherical harmonic coefficients of this series are determined once and then frequently used in different geosciences disciplines. The type of the observations, namely, terrestrial and/or satellite measurements, plays an important role in determining these coefficients. In satellite gravity gradiometry (SGG), the second-order derivatives of the Earth gravitational potential is measured. Because of the high sensitivity of the SGG data to the Earth's gravitational field, it is possible to obtain a high-degree pure satellite Earth gravitational model using these data-i.e., up to a degree and order of 300. There are (at least) two approaches for computing the geopotential coefficients using the SGG data in the space-wise approach (see, for example, Koop, 1993; Rummel et al., 1993; Sharifi, 2006): the least-squares solution or quadrature formulas. By taking advantage of the orthogonality of the spherical harmonic, it is possible to construct integral formulas to determine the geopotential coefficients. However, the solution of such integrals will not be optimal for the harmonic coefficients. In order to use integral formulas for solving gradiometric boundary value problems (GBVP), Gelderen and Rummel (2001, 2002) presented three combinations for five independent components of the gravitational gradients' tensor. Martinec

Copyright (C) The Society of Geomagnetism and Earth, Planetary and Space Sciences (SGEPSS); The Seismological Society of Japan; The Volcanological Society of Japan; The Geodetic Society of Japan; The Japanese Society for Planetary Sciences; TERRAPUB.

doi:10.5047/eps.2010.01.002
(2003) solved the GBVP in the spectral domain to reveal explicit relations between geopotential coefficients and these three integral formulas. The integrands of these integrals are combinations of the gravitational gradients and tensor spherical harmonics (which are globally orthogonal). Each integral delivers a set of solutions for geopotential coefficients, and it is clear that they are not optimal solutions because of the lack of redundancy. Gelderen and Rummel (2001, 2002) used the least-squares solution for these three integrals, ultimately concluding that the weighted mean can be a good solution for the spectral combination. Other researchers have also worked on the combined solution, but all used the least-squares solution of the geopotential coefficients directly by the Gauss-Markov model and did not take advantage the solution of the GBVP (see, for example, Kusche, 2003; Grafarend, 2006; Xu et al., 2006, 2007).

Sjöberg $(1980,1981)$ and Wenzel (1981) investigated the spectral combination, but their methods are very general. Sjöberg (1984a) then developed this technique to a spectral form of the Stokes integral and presented his least-squares modification of Stokes' formula. However, the main idea of his work comes from Bjerhammar (1983) who suggested using variance component estimation (VCE) to mix discrete boundary value problems; however, combining the spectral solutions of GBVP is totally different. Bjerhammar (1983) used this technique to mix solutions of the Fredholm integral equations of the first kind, but in the case of gradiometry, he used the spectral solutions of the GBVP. Here, an attempt is made to estimate an optimal value for each spectrum. Using this approach, we use the condition adjustment model (CAM) and integrate the benefits of the VCE pro- 
cess to obtain a better optimal spectral combination. This approach has not been considered until now. The strategy which is presented in this paper is not restricted just to the GBVP, and it can be used for other aspects of the spectral combination, such as the combination of other solutions of geodetic boundary value problems.

The spectral solution of the GBVP is presented in Section 2. Section 3 presents different simple methods of the optimal spectral combination, and the VCE is quickly reviewed and the degree-order variance component (DOVC) are defined and formulated according to the GBVP in Section 4. The formulas are also discussed in theoretical and practical terms in Section 4. The conclusions are presented in Section 5.

\section{Spectral Solution of the GBVP}

Three independent combinations of the gradiometric tensor were considered in the solution of the GBVP by Gelderen and Rummel (2001, 2002). This combination is made in such a way that we can take advantage of the orthogonality of the tensor spherical harmonics. Since three combinations are considered, there will be three solutions for the geopotential coefficients. These solutions are (the solutions are presented without proof; the interested reader is referred to, for example, Martinec, 2003):

$$
\begin{aligned}
t_{n m}^{(1)}= & \frac{R^{3} / 4 \pi G M}{(n+1)(n+2)}\left(\frac{r}{R}\right)^{n+3} \iint_{\sigma} T_{z z}(Q) Y_{n m}(Q) d \sigma,(1 \mathrm{a}) \\
t_{n m}^{(2)}= & -\frac{R^{3} / 4 \pi G M}{n(n+1)(n+2)}\left(\frac{r}{R}\right)^{n+3} \iint_{\sigma}\left[T_{x z}(Q) E_{n m}(Q)\right. \\
& \left.+T_{y z}(Q) F_{n m}(Q)\right] d \sigma \\
t_{n m}^{(3)}= & \frac{R^{3} / 4 \pi G M}{(n-1) n(n+1)(n+2)}\left(\frac{r}{R}\right)^{n+3} \iint_{\sigma}\left\{\left[T_{x x}(Q)\right.\right. \\
& \left.\left.-T_{y y}(Q)\right] G_{n m}(Q)-2 T_{x y}(Q) H_{n m}(Q)\right\} d \sigma
\end{aligned}
$$

where $t_{n m}^{(1)}, t_{n m}^{(2)}$ and $t_{n m}^{(3)}$ are the geopotential coefficients according to first, second and third combinations of the gravitational gradients, respectively. $T_{z z}(Q), T_{x x}(Q), T_{y y}(Q)$, $T_{x y}(Q), T_{x z}(Q)$ and $T_{y z}(Q)$ are the elements of the gradiometric tensor at integration point $Q, R$ is the mean radius of the Earth, $r$ is the geocentric radius of the computation point $P$ (in satellite gradiometry, it is $R+$ altitutde of satellite), $\sigma$ is the unit sphere on which the integration is taken, and $d \sigma$ is the integration element. $Y_{n m}(Q)$ is the scalar spherical harmonic of degree $n$ and order $m$

$$
\begin{aligned}
E_{n m}(Q) & =\frac{\partial Y_{n m}(Q)}{\partial \theta} \\
F_{n m}(Q) & =\frac{\partial Y_{n m}(Q)}{(\sin \theta \partial \lambda)} \\
G_{n m}(Q) & =\left[\frac{\partial^{2}}{\partial \theta^{2}}-\cot \theta \frac{\partial}{\partial \theta}-\frac{\partial^{2}}{\left(\sin ^{2} \theta \partial \lambda^{2}\right)}\right] Y_{n m}(Q),
\end{aligned}
$$

and

$$
H_{n m}(Q)=2 \frac{\partial}{\partial \theta}\left[\frac{\partial Y_{n m}(Q)}{(\sin \theta \partial \lambda)}\right] .
$$

Equations (2a)-(2d) are related to the definition of the tensor spherical harmonics. Let us vectorize Eqs. (1a)-(1c) in the following way:

$$
\begin{aligned}
t_{n m}^{(1)} & =\mathbf{b}_{z z}^{n m} \mathbf{t}_{z z}, \\
t_{n m}^{(2)} & =\mathbf{b}_{x z}^{n m} \mathbf{t}_{x z}+\mathbf{b}_{y z}^{n m} \mathbf{t}_{y z}, \\
t_{n m}^{(3)} & =\mathbf{b}_{x x}^{n m} \mathbf{t}_{x x}+\mathbf{b}_{y y}^{n m} \mathbf{t}_{y y}+\mathbf{b}_{x y}^{n m} \mathbf{t}_{x y} .
\end{aligned}
$$

where $\mathbf{b}_{z z}^{n m}, \mathbf{b}_{x x}^{n m}, \mathbf{b}_{y y}^{n m}, \mathbf{b}_{x y}^{n m}, \mathbf{b}_{x z}^{n m}$, and $\mathbf{b}_{y z}^{n m}$ are row vectors obtained by discretizing Eqs. (1a)-(1c) and $\mathbf{t}_{z z}, \mathbf{t}_{x x}, \mathbf{t}_{y y}, \mathbf{t}_{x y}$, $\mathbf{t}_{x z}$ and $\mathbf{t}_{y z}$ are the column vectors of the $T_{z z}(Q), T_{x x}(Q)$, $T_{y y}(Q), T_{x y}(Q), T_{x z}(Q)$ and $T_{y z}(Q)$ observations, respectively. In fact, the integrals are converted to a simple inner product of the discretized integral vector (functional) and observation vectors. By using such a vectorized model, it is easy to use the error propagation law to estimate the error of each geopotential coefficient at degree $n$ and order $m$. For example, uncorrelated observations are assumed, we obtain:

$$
\begin{aligned}
\sigma_{t_{n m}^{(1)}} & = \pm \sqrt{\mathbf{b}_{z z}^{n m}\left(\mathbf{b}_{z z}^{n m}\right)^{T}} \sigma_{\mathbf{t}_{z z}} \\
\sigma_{t_{n m}^{(2)}} & = \pm \sqrt{\mathbf{b}_{x z}^{n m}\left(\mathbf{b}_{x z}^{n m}\right)^{T} \sigma_{\mathbf{t}_{x z}}^{2}+\mathbf{b}_{y z}^{n m}\left(\mathbf{b}_{y z}^{n m}\right)^{T} \sigma_{\mathbf{t}_{y z}}^{2}} \\
\sigma_{t_{n m}^{(3)}} & = \pm \sqrt{\mathbf{b}_{x x}^{n m}\left(\mathbf{b}_{x x}^{n m}\right)^{T} \sigma_{\mathbf{t}_{x x}}^{2}+\mathbf{b}_{y y}^{n m}\left(\mathbf{b}_{y y}^{n m}\right)^{T} \sigma_{\mathbf{t}_{y y}}^{2}+\mathbf{b}_{x y}^{n m}\left(\mathbf{b}_{x y}^{n m}\right)^{T} \sigma_{\mathbf{t}_{x y}}^{2}}
\end{aligned}
$$

where $\sigma_{\mathbf{t}_{z z}}, \sigma_{\mathbf{t}_{x z}}, \sigma_{\mathbf{t}_{y z}}, \sigma_{\mathbf{t}_{x x}}, \sigma_{\mathbf{t}_{y y}}$ and $\sigma_{\mathbf{t}_{x y}}$ are the standard error of the SGG data.

The errors are propagated according to Eqs. (3a)-(3c), which are discretized integral solutions. The higher the data resolution, the lower the discretization error and the higher the degree of the achievable solution (Rummel, 1997). Actually, the degree of resolution shows the maximum acceptable truncation for the spherical harmonic analysis, and it is not meaningful to estimate higher degrees of geopotential coefficients than this degree; see also Albertella et al. (1993) and Sneeuw (2000). This could therefore be one criterion for the maximum degree of the geopotential model being computed from the SGG data. Theoretically, the integral formulas are perfect and able to continue the SGG data down to sea level using spherical harmonics for all frequencies. However, in practice, this is not true as the integrals should be discretized and solved. Discretization of these integrals is in accordance with the resolution of the available SGG data, and continuous coverage for the SGG data is never achieved, restricting us to a certain resolution. The degree of resolution could be a criterion for cutting the higher frequencies according to the SGG data resolution.

The gradiometric tensor has five independent elements, which are measured at satellite level. By using combinations of these observable elements, we can obtain three different sets of geopotential coefficients. As mentioned above, solutions of the three GBVP do not yield the same results in practice. The main explanation for these differences may be a dependence on the different behaviour of the discretized integrals and noise contaminated with real data. In reality, we cannot come to the same results by these three solutions. We already know that neither of the solutions is optimal, which is why some research groups are trying to use the least-squares approach to compute a geopotential model: the latter is an optimal estimator, and overdetermination helps the solution to suppress noise in the 
observations. After a certain number of observations, we will have redundancy in the solution, and the least-squares method is beneficial.

\section{Simple Approaches to Combine the GBVP So- lutions}

In this section we present a number of simple approaches to combining the spectral solutions in SGG. Specifically, these are the (1) simple mean and weighted mean and (2) a CAM to derive an optimal spectral combination.

\subsection{Simple mean and weighted mean}

Gelderen and Rummel (2001, 2002) investigated the least-squares solutions of the GBVP and finally came to the conclusion that the weighted mean is one way of combining these three solutions. Rummel et al. (1993) made similar statements on this subject (see also Sanso and Sona, 1995).

In order to test the spectral combination based on the simple and weighted means, let us consider $\sigma_{\mathbf{t}_{z z}}=\sigma_{\mathbf{t}_{x z}}=\sigma_{\mathbf{t}_{y z}}=$ $\sigma_{\mathbf{t}_{x x}}=\sigma_{\mathbf{t}_{y y}}=\sigma_{\mathbf{t}_{x y}}=0.01 \mathrm{E}$ and the resolution $30^{\prime} \times 30^{\prime}$ for the SGG data at the 250-km level. The EGM96 geopotential model (Lemoine et al., 1998) has been used to synthesize the SGG data at the satellite level and analyse the gravitational field using the descretized integrals of Eqs. (1a)-(1c). Also, EGM96 is supposed to be the true solution in this study. The degree of resolution for the grid of the SGG data with $30^{\prime} \times 30^{\prime}$ resolution at the $250-\mathrm{km}$ level is about 198 , 199 and 230 for the horizontal-horizontal (HH), verticalhorizontal (VH) and vertical-vertical (VV) solutions, respectively. The error of the each geopotential coefficient is estimated using the error propagation law, Eqs. (4a)-(4c). The reciprocal squared errors are considered as the weight for each coefficient. Figure 1 shows the simple mean and weighted mean solutions to the spectral combinations.

As it can be seen, the simple mean and the weighted mean yield more or less the same results in the presence of the discretization error; the spectral differences are visualized in Fig. 1(c). Even the error degree variances of these solutions are more or less the same. Consequently, in the presence of only the discretization error, we can say that both averaging processes deliver about the same results for the degrees between 200 and 250. The results of these solutions will differ if random noise is considered in the SGG data. In the following subsection, we report on another approach for the spectral combination of the GBVP in an attempt to obtain better results. To this end, we first review the adjustment of condition model.

\subsection{The CAM}

A quick review of the CAM follows:

$$
\mathbf{B}(\mathbf{L}-\boldsymbol{\varepsilon})=\mathbf{w}=\mathbf{c}^{\prime}-\mathbf{B} \mathbf{L} \text { with } E\left\{\boldsymbol{\varepsilon} \boldsymbol{\varepsilon}^{T}\right\}=\sigma_{0}^{2} \mathbf{Q},
$$

where $\mathbf{B}$ is the coefficients matrix of observations, $\boldsymbol{\varepsilon}$ stands for the vector of observation noise, $\mathbf{w}$ is the misclousure vector, $E\{$.$\} stands for the mathematical expectation, \mathbf{Q}$ is the co-factor matrix or variance-covariance matrix of the observations, $\mathbf{c}^{\prime}$ is the constant vector of the CAM, $\mathbf{L}$ is the observation vector and, finally, $\sigma_{0}^{2}$ is an a priori variance factor. In general, the least-squares estimate of the residuals will be

$$
\hat{\boldsymbol{\varepsilon}}=\mathbf{B}_{\mathbf{Q O}}^{-} \mathbf{W}
$$
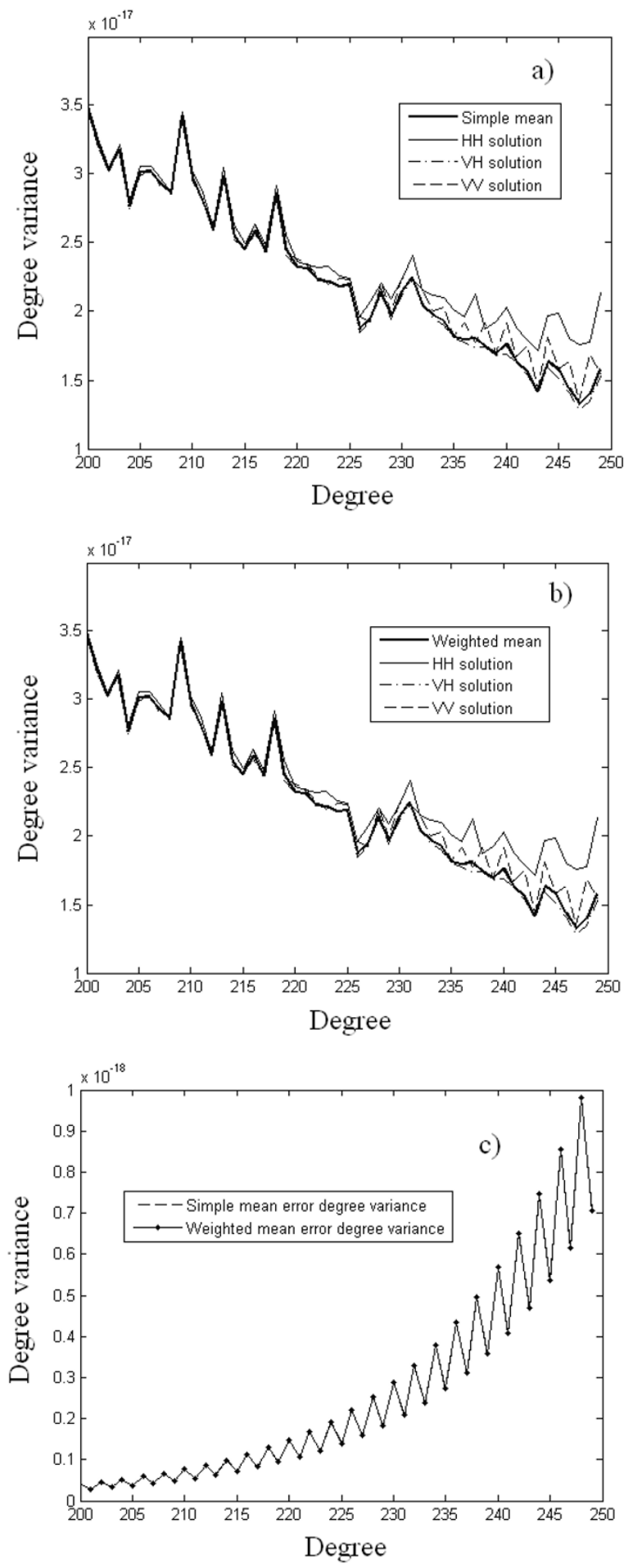

Fig. 1. (a) Simple mean solution, (b) weighted mean, (c) error degree variance of simple and weighted means.

where $\mathbf{B}_{\mathbf{Q} \mathbf{O}}^{-}=\mathbf{Q B}^{T}\left(\mathbf{B Q} \mathbf{B}^{T}\right)^{-}$is a left (normal) inverse of the matrix $\mathbf{B}$; the minus sign superscripted over the parenthesis stands for the generalized inverse (Bjerhammar, 1973).

Considering Eqs. (3a)-(3c), we can write two CAMs, if the $\mathbf{B}$ and $\mathbf{L}$ matrices have the following form

$$
\mathbf{B}^{n m}(\mathbf{L}-\boldsymbol{\varepsilon})=0,
$$


where

$$
\begin{aligned}
& \mathbf{L}=\left[\begin{array}{llllll}
\mathbf{t}_{z z} & \mathbf{t}_{x x} & \mathbf{t}_{y y} & \mathbf{t}_{x y} & \mathbf{t}_{x z} & \mathbf{t}_{y z}
\end{array}\right]^{T}, \\
& \mathbf{B}^{n m}=\left[\begin{array}{cccccc}
\mathbf{b}_{z z}^{n m} & -\mathbf{b}_{x x}^{n m} & -\mathbf{b}_{y y}^{n m} & -\mathbf{b}_{x y}^{n m} & 0 & 0 \\
\mathbf{b}_{z z}^{n m} & 0 & 0 & 0 & -\mathbf{b}_{x z}^{n m} & -\mathbf{b}_{y z}^{n m}
\end{array}\right] \text {. }
\end{aligned}
$$

Also, if we assume no correlation between different types of observations, the co-factor matrix will have a block diagonal structure:

$$
\mathbf{Q}=\operatorname{diag}\left[\begin{array}{llllll}
\mathbf{Q}_{z z} & \mathbf{Q}_{x x} & \mathbf{Q}_{y y} & \mathbf{Q}_{x y} & \mathbf{Q}_{x z} & \mathbf{Q}_{y z}
\end{array}\right] .
$$

where $\mathbf{Q}_{z z}, \mathbf{Q}_{x x}, \mathbf{Q}_{y y}, \mathbf{Q}_{x y}, \mathbf{Q}_{x z}$ and $\mathbf{Q}_{y z}$ are the cofactor matrices of $\mathbf{t}_{z z}, \mathbf{t}_{x x}, \mathbf{t}_{y y}, \mathbf{t}_{x y}, \mathbf{t}_{x z}$ and $\mathbf{t}_{y z}$, respectively. We also consider $\mathbf{C}^{n m}=\mathbf{B}^{n m} \mathbf{Q}\left(\mathbf{B}^{n m}\right)^{T}$. The superscript $n, m$ means that the element values change by degree $n$ and order $m$. The rows of $\mathbf{B}^{n m}$ is always equal to 2 , but the number of columns depends on the resolution of the analysis and descretized integrals. Subsequently, $\mathbf{C}^{n m}$ is a $2 \times 2$ matrix and independent on the SGG data. The elements of $\mathbf{C}^{n m}$ are:

$$
\begin{aligned}
c_{11}^{n m}= & \mathbf{b}_{z z}^{n m} \mathbf{Q}_{z z}\left(\mathbf{b}_{z z}^{n m}\right)^{T}+\mathbf{b}_{x x}^{n m} \mathbf{Q}_{x x}\left(\mathbf{b}_{x x}^{n m}\right)^{T} \\
& +\mathbf{b}_{y y}^{n m} \mathbf{Q}_{y y}\left(\mathbf{b}_{y y}^{n m}\right)^{T}+\mathbf{b}_{x y}^{n m} \mathbf{Q}_{x y}\left(\mathbf{b}_{x y}^{n m}\right)^{T}, \\
c_{22}^{n m}= & \mathbf{b}_{z z}^{n m} \mathbf{Q}_{z z}\left(\mathbf{b}_{z z}^{n m}\right)^{T}+\mathbf{b}_{x z}^{n m} \mathbf{Q}_{x z}\left(\mathbf{b}_{x z}^{n m}\right)^{T} \\
& +\mathbf{b}_{y z}^{n m} \mathbf{Q}_{y z}\left(\mathbf{b}_{y z}^{n m}\right)^{T}, \\
c_{21}^{n m}= & c_{12}^{n m}=\mathbf{b}_{z z}^{n m} \mathbf{Q}_{z z}\left(\mathbf{b}_{z z}^{n m}\right)^{T} .
\end{aligned}
$$

Similarly, the mis-closure vector is a $2 \times 1$ vector with elements (where $\mathbf{c}^{\prime}=0$ ):

$\mathbf{w}^{n m}=\left[\begin{array}{c}w_{1}^{n m} \\ w_{2}^{n m}\end{array}\right]=\left[\begin{array}{l}-\mathbf{b}_{z z}^{n m} \mathbf{t}_{z z}^{T}+\mathbf{b}_{x x}^{n m} \mathbf{t}_{x x}^{T}+\mathbf{b}_{y y}^{n m} \mathbf{t}_{y y}^{T}+\mathbf{b}_{x y}^{n m} \mathbf{t}_{x y}^{T} \\ -\mathbf{b}_{z z}^{n m} \mathbf{t}_{z z}^{T}+\mathbf{b}_{x z}^{n m} \mathbf{t}_{x z}^{T}+\mathbf{b}_{y z}^{n m} \mathbf{t}_{y z}^{T}\end{array}\right]$.

Following the substitution of Eqs. (9a)-(9c) and (10) into Eq. (6), then a further simplification, the adjusted error vector is obtained:

$$
\left[\begin{array}{c}
\hat{\boldsymbol{\varepsilon}}_{z z}^{n m} \\
\hat{\boldsymbol{\varepsilon}}_{x x}^{n m} \\
\hat{\boldsymbol{\varepsilon}}_{y y}^{n m} \\
\hat{\boldsymbol{\varepsilon}}_{x y}^{n m} \\
\hat{\boldsymbol{\varepsilon}}_{x z}^{n m} \\
\hat{\boldsymbol{\varepsilon}}_{y z}^{n m}
\end{array}\right]=\frac{1}{\left|\mathbf{C}^{n m}\right|}\left[\begin{array}{c}
\mathbf{Q}_{z z}\left(\mathbf{b}_{z z}^{n m}\right)^{T}\left(c_{22}^{n m} w_{1}^{n m}-c_{12}^{n m} w_{2}^{n m}\right) \\
+\mathbf{Q}_{z z}\left(\mathbf{b}_{z z}^{n m}\right)^{T}\left(c_{11}^{n m} w_{2}^{n m}-c_{21}^{n m} w_{1}^{n m}\right) \\
-\mathbf{Q}_{x x}\left(\mathbf{b}_{x x}^{n m}\right)^{T}\left(c_{22}^{n m} w_{1}^{n m}-c_{12}^{n m} w_{2}^{n m}\right) \\
-\mathbf{Q}_{y y}\left(\mathbf{b}_{y y}^{n m}\right)^{T}\left(c_{22}^{n m} w_{1}^{n m}-c_{12}^{n m} w_{2}^{n m}\right) \\
-\mathbf{Q}_{x y}\left(\mathbf{b}_{x y}^{n m}\right)^{T}\left(c_{22}^{n m} w_{1}^{n m}-c_{12}^{n m} w_{2}^{n m}\right) \\
-\mathbf{Q}_{x z}\left(\mathbf{b}_{x z}^{n m}\right)^{T}\left(c_{11}^{n m} w_{2}^{n m}-c_{21}^{n m} w_{1}^{n m}\right) \\
-\mathbf{Q}_{y z}\left(\mathbf{b}_{y z}^{n m}\right)^{T}\left(c_{11}^{n m} w_{2}^{n m}-c_{21}^{n m} w_{1}^{n m}\right)
\end{array}\right]
$$

where $|\cdot|$ stands for determinant operation.

The main difference between the adjustment of the CAM and the Gauss-Markov model is the computation of the adjusted residual vector before estimation of the unknown parameters. After correction of the observations based on the obtained residuals, all three integral solutions must deliver the same results (i.e. the geopotential coefficients of degree
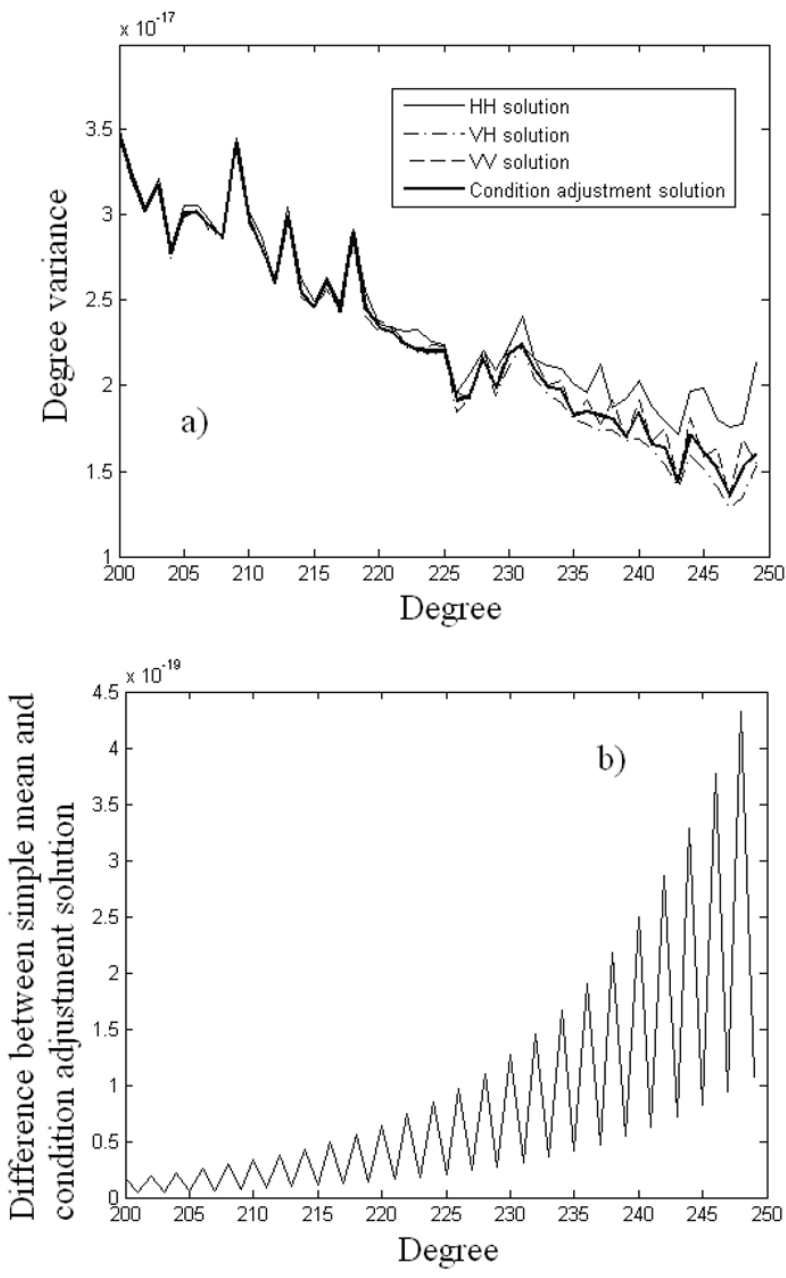

Fig. 2. (a) Condition adjustment solution. (b) Difference between the condition adjustment solution and simple mean.

$n$ and order $m$ ). As it does not matter which integral is used to compute the coefficients, one can use the simplest integral, or in other words, the VV solution of the GBVP (Eq. (1a) is preferred)

$$
\hat{\mathbf{L}}=\mathbf{L}-\hat{\boldsymbol{\varepsilon}}
$$

and using the well-known error propagation laws, we can estimate the error of the corrected observations (Eshagh, 2009):

$$
\begin{aligned}
\mathbf{Q}_{\hat{\mathbf{L}}} & =\mathbf{Q}_{\mathbf{L}}+\mathbf{Q}_{\hat{\boldsymbol{\varepsilon}}} \\
& =\mathbf{b}^{n m} \mathbf{Q}\left(\mathbf{b}^{n m}\right)^{T}+\mathbf{Q}\left(\mathbf{b}^{n m}\right)^{T}\left[\mathbf{b}^{n m} \mathbf{Q}\left(\mathbf{b}^{n m}\right)^{T}\right]^{-} \mathbf{b}^{n m} \mathbf{Q},
\end{aligned}
$$

where, $\mathbf{Q}_{\hat{\mathbf{L}}}$ is the co-factor matrix of the corrected observations, $\mathbf{Q}_{\mathbf{L}}$ is the co-factor matrix of the observations and $\mathbf{Q}_{\hat{\boldsymbol{\varepsilon}}}$ is for the estimated residuals (the corrections).

Figure 2 shows the condition adjustment solution of three GBVP.

Figure 2(a) shows the condition adjustment solution of the three GBVP. A comparison of this figure with Fig. 1(a) and 1 (b) reveals that the solutions are again very similar, as was expected, because in both cases, namely, the simple 
mean and the condition adjustment solution, the quadratic form of the residual vector is minimized. The difference between the condition solution and the simple mean is provided in Fig. 2(b). The differences are very small, both compared to the signal itself (see Fig. 2(a)) and in order of numerical integration error. The question is: why should we use the complicated solution of the CAM, while the simple mean practically provides the same results and is extremely simple? The answer is related to the fact that although both solutions yield more or less the same results, the CAM opens another way to combine these solutions. The idea is not just to use the CAM to combine the solutions of the GBVP in a simple way, but we are actually seeking a way to re-weight the observations to obtain a better spectral combination. The CAM provides this by considering the VCE technique. In Section 4, we review the concept of the VCE and explain how to use the VCE in spectral combination of the GBVP solutions.

\section{VCE and Re-weighting Solution}

Another method of combination is to use the VCE and re-weight the observations to obtain optimal value for the geopotential coefficients. The CAM is not sensitive to an a priori variance factor. The only important matter in the CAM is to have different accuracies. Each CAM has a misclosure vector which can be used to determine the variance components (VCs) according to a pre-described stochastic model for the observations error. In fact, in the VCE, an a posteriori variance factor is estimated for each set of observations. The observation weights are re-scaled in order to obtain a better match between the residuals and misclosures. In the following sections we summarize wellknown methods of the VCE, namely the best quadratic unbiased estimation (BQUE) and best quadratic unbiased non-negative estimation (BQUNE) (Sjöberg, 1984b) and the modified best quadratic unbiased non-negative estimator (MBQUNE) (Eshagh and Sjöberg, 2008). The discussion is opened with the Gauss-Helmert model, followed by the special case of the CAM. Degree-order VCs (DOVC) are defined as a VC that is estimated according to the degree and order of the solution.

\subsection{The Gauss-Helmert model}

The Gauss-Helmert model is a generalized model of the Gauss-Markov and the CAM, i.e. these models are special cases of the Gauss-Helmert model. This model has the following form:

$$
\mathbf{A x}+\mathbf{B} \boldsymbol{\varepsilon}=\mathbf{w}, \text { with } E\left\{\boldsymbol{\varepsilon} \varepsilon^{T}\right\}=\mathbf{Q}, \text { and } E\{\boldsymbol{\varepsilon}\}=0,
$$

where, $\mathbf{A}$ and $\mathbf{B}$ are the first and second design matrices of dimensions $(k \times m)$ and $(k \times n)$, respectively, with $n \geq k \geq$ $m, \mathbf{x}$ is the vector of unknowns, $\boldsymbol{\varepsilon}$ is a residual vector, $\mathbf{w}$ is the vector of mis-closures, $\mathbf{Q}$ is the covariance matrix of observations and $E\{\cdot\}$ stands for the statistical expectation. We assume that the variance-covariance model is

$$
\mathbf{Q}=\sum_{i=1}^{q} \sigma_{i}^{2} \mathbf{Q}_{i}
$$

where $\mathbf{Q}_{i}$ are known positive semi-definite sub-matrices of $\mathbf{Q}$, and $\sigma_{i}^{2}$ are the unknown VCs. The above equation can be converted to the CAM as (Eshagh, 2009):

$$
\left(\mathbf{I}-\mathbf{A}^{0}\right) \mathbf{B} \boldsymbol{\varepsilon}=\left(\mathbf{I}-\mathbf{A}^{0}\right) \mathbf{w}, \text { or } \overline{\mathbf{B}} \boldsymbol{\varepsilon}=\overline{\mathbf{w}},
$$

where, $\mathbf{A}^{0}=\mathbf{A} \mathbf{A}^{-}=\mathbf{A}\left(\mathbf{A}^{T} \mathbf{C}^{-} \mathbf{A}\right)^{-} \mathbf{A}^{T} \mathbf{C}^{-}$. Hence, $\mathbf{A}^{-}$ stands for a specific generalized inverse of $\mathbf{A}$, namely $\mathbf{A}^{-}=$ $\left(\mathbf{A}^{T} \mathbf{C}^{-} \mathbf{A}\right)^{-} \mathbf{A}^{T} \mathbf{C}^{-}$, where

$$
\mathbf{C}=\sum_{i=1}^{q} \sigma_{i}^{2} \mathbf{C}_{i} \text { and } \mathbf{C}_{i}=\mathbf{B} \mathbf{Q}_{i} \mathbf{B}^{T},(\operatorname{rank}(\mathbf{C})=k)
$$

Alternatively, we obtain the CAM if $\mathbf{A}=0$ in Eq. (13). Similarly, the method of adjustment by elements (or GaussMarkov model) is obtained if $\mathbf{B}=\mathbf{I}$ and $\mathbf{w}=\mathbf{L}$.

\subsection{The BQUE of a DOVC}

Now, suppose that we want to estimate a linear combination $\mathbf{p}^{T} \boldsymbol{\sigma}=\sum_{i=1}^{q} p_{i} \sigma_{i}^{2}$ of the VCs related with Eq. (13), where $p_{i}$ are arbitrary coefficients. A sufficient condition for unbiasedness of the estimator $\overline{\mathbf{w}}^{T} \mathbf{M} \overline{\mathbf{w}}$ of $\mathbf{p}^{T} \boldsymbol{\sigma}$ is that (Rao, 1971; Sjöberg, 1983; Rao and Kleffe, 1988)

$$
p_{i}=\operatorname{trace}\left(\mathbf{M K}_{i}\right)
$$

where

$$
\mathbf{K}_{i}=\left(\mathbf{I}-\mathbf{A}^{0}\right) \mathbf{C}_{i}\left(\mathbf{I}-\mathbf{A}^{0}\right)^{T},
$$

and, in the case of normally distributed observation errors, the BQUE, and, in the general case, the MInimum Norm Quadratic Unbiased Estimator (MINQUE) of $\mathbf{p}^{T} \boldsymbol{\sigma}$ is provided by $\mathbf{p}^{T} \mathbf{S}^{-1} \mathbf{u}$ and

$$
\begin{aligned}
s_{i j} & =\operatorname{trace}\left(\mathbf{R C}_{i} \mathbf{R C}_{j}\right) ; \quad i, j=1,2, \ldots, q \\
u_{i} & =\overline{\mathbf{w}}^{T} \mathbf{R} \mathbf{C}_{i} \mathbf{R} \overline{\mathbf{w}} ; \quad i=1,2, \ldots, q \\
\mathbf{R} & =\mathbf{C}^{-1}\left(\mathbf{I}-\mathbf{A}^{0}\right)=\left(\mathbf{I}-\mathbf{A}^{0}\right)^{T} \mathbf{C}^{-1} .
\end{aligned}
$$

Proposition 1 The BQUE of the DOVC based on Eq. (7) with stochastic model 1

$\mathbf{Q}=\sigma_{\mathrm{VV}}^{2} \mathbf{Q}_{\mathrm{VV}}+\sigma_{\mathrm{HH}}^{2} \mathbf{Q}_{\mathrm{HH}}+\sigma_{\mathrm{VH}}^{2} \mathbf{Q}_{\mathrm{VH}}($ stochastic model 1) where

$$
\begin{aligned}
& \mathbf{Q}_{\mathrm{vV}}=\operatorname{diag}\left(\begin{array}{lllllll}
\mathbf{Q}_{z z} & 0 & 0 & 0 & 0 & 0
\end{array}\right) \\
& \mathbf{Q}_{\mathrm{HH}}=\operatorname{diag}\left(\begin{array}{lllllll}
0 & \mathbf{Q}_{x x} & \mathbf{Q}_{y y} & \mathbf{Q}_{z z} & 0 & 0
\end{array}\right) \\
& \mathbf{Q}_{\mathrm{VH}}=\operatorname{diag}\left(\begin{array}{lllllll}
0 & 0 & 0 & 0 & \mathbf{Q}_{x z} & \mathbf{Q}_{y z}
\end{array}\right)
\end{aligned}
$$

is:

$$
\begin{aligned}
& \left(\hat{\sigma}_{j}^{2}\right)^{n m}=\frac{\left[\left(w_{1}^{n m}\right)^{2}+\left(w_{2}^{n m}\right)^{2}\right]\left[\left(c_{22}^{n m}\right)^{2}+\left(c_{12}^{n m}\right)^{2}\right]-2 w_{1}^{n m} w_{2}^{n m}\left(c_{22}^{n m}+c_{11}^{n m}\right) c_{12}^{n m}}{k_{j}^{n m}\left[\left(c_{22}^{n m}\right)^{2}+2\left(c_{12}^{n m}\right)^{2}+\left(c_{11}^{n m}\right)^{2}\right]} \\
& j=\mathrm{VV}, \mathrm{VH}, \text { and HH }
\end{aligned}
$$

Proof. As previously mentioned, the VCs are determined unbiasedly by $\mathbf{p}^{T} \mathbf{S}^{-1} \mathbf{u}$, but in the CAM, the elements of the coefficients matrix $\mathbf{S}$ are:

$$
s_{i j}=\operatorname{trace}\left[\left(\mathbf{K}^{n m}\right)^{-1} \mathbf{K}_{i}^{n m}\left(\mathbf{K}^{n m}\right)^{-1} \mathbf{K}_{j}^{n m}\right]
$$

where $i, j=\mathrm{VV}, \mathrm{VH}$ and $\mathrm{HH}$ corresponding to 1,2 are 3 as row and columns number, respectively, and the right-hand side vector elements are:

$$
u_{i}=\left(\mathbf{w}^{n m}\right)^{T}\left(\mathbf{K}^{n m}\right)^{-1} k_{i}^{n m}\left(\mathbf{K}^{n m}\right)^{-1} \mathbf{w}^{n m},
$$


where $k_{i}^{n m}, i=\mathrm{VV}, \mathrm{VH}$ and $\mathrm{HH}$ are:

$$
\begin{aligned}
k_{\mathrm{VV}}^{n m}= & \mathbf{b}_{z z}^{n m} \mathbf{Q}_{z z}\left(\mathbf{b}_{z z}^{n m}\right)^{T}, \\
k_{\mathrm{HH}}^{n m}= & \mathbf{b}_{x x}^{n m} \mathbf{Q}_{x x}\left(\mathbf{b}_{x x}^{n m}\right)^{T}+\mathbf{b}_{y y}^{n m} \mathbf{Q}_{y y}\left(\mathbf{b}_{y y}^{n m}\right)^{T} \\
& +\mathbf{b}_{x y}^{n m} \mathbf{Q}_{x y}\left(\mathbf{b}_{x y}^{n m}\right)^{T}, \\
k_{\mathrm{VH}}^{n m}= & \mathbf{b}_{x z}^{n m} \mathbf{Q}_{x z}\left(\mathbf{b}_{x z}^{n m}\right)^{T}+\mathbf{b}_{y z}^{n m} \mathbf{Q}_{y z}\left(\mathbf{b}_{y z}^{n m}\right)^{T},
\end{aligned}
$$

which are derived according to Eq. (17b). Substituting Eqs. (21a)-(21c) into Eq. (19) results in

$$
s_{i j}=\frac{k_{i}^{n m} k_{j}^{n m}\left[\left(c_{22}^{n m}\right)^{2}+2\left(c_{12}^{n m}\right)^{2}+\left(c_{11}^{n m}\right)^{2}\right]}{\left|\mathbf{C}^{n m}\right|^{2}} .
$$

Also, by substituting Eqs. (21a)-(21c) into Eq. (20), we obtain

$$
\begin{aligned}
u_{i}= & \frac{k_{i}^{n m}}{\left|\mathbf{C}^{n m}\right|^{2}}\left\{\left(w_{1}^{n m}\right)^{2}\left[\left(c_{22}^{n m}\right)^{2}+\left(c_{12}^{n m}\right)^{2}\right]\right. \\
& -2 w_{1}^{n m} w_{2}^{n m} c_{21}^{n m}\left(c_{22}^{n m}+c_{11}^{n m}\right) \\
& \left.+\left(w_{2}^{n m}\right)^{2}\left[\left(c_{11}^{n m}\right)^{2}+\left(c_{12}^{n m}\right)^{2}\right]\right\}
\end{aligned}
$$

By inserting Eqs. (23) and (22) into $\mathbf{p}^{T} \mathbf{S}^{-1} \mathbf{u}$ and making some simplifications, the proposition is proven.

The DOVCs of the gravitational gradients can be estimated using Proposition 1 based on stochastic model 1. As the proposition shows, the numerator of this estimator is independent of the form of the stochastic model. The only parameter that depends on the stochastic model is $k_{j}$ (corresponding to each $\left(\hat{\sigma}_{j}^{2}\right)^{n m}$ (DOVC)). In this combination approach for each geopotential coefficient with degree $n$ and order $m$, three DOVCs are estimated; thus, the estimated DOVCs differ by degrees and orders.

According to Proposition 1, the denominator of the proposition is always positive. Here, the only possible way for the DOVC to come out negative is to have a negative numerator. The first term of the numerator is also always positive; thus, we can write the following condition to obtain negative DOVCs:

$$
\frac{\left[\left(w_{1}^{n m}\right)^{2}+\left(w_{2}^{n m}\right)^{2}\right]\left[\left(c_{22}^{n m}\right)^{2}+\left(c_{12}^{n m}\right)^{2}\right]}{2\left(c_{22}^{n m}+c_{11}^{n m}\right) c_{12}^{n m}}<w_{1}^{n m} w_{2}^{n m}
$$

The left-hand side of this inequality is always positive, but the right-hand side will be positive if either $w_{1}, w_{2}<0$ or $w_{1}, w_{2}>0$. When the positive value $w_{1} w_{2}$ is larger than the left-hand side of Eq. (24), negative DOVCs are obtained. Positive DOVCs are obtained when $w_{1}$ and $w_{2}$ have opposite signs.

Corollary 1 The BQUE of the DOVC based on Eq. (7) and stochastic model 2

$\mathbf{Q}=\sigma_{z z}^{2} \mathbf{Q}_{z z}^{\prime}+\sigma_{x x}^{2} \mathbf{Q}_{x x}^{\prime}+\sigma_{y y}^{2} \mathbf{Q}_{y y}^{\prime}+\sigma_{x y}^{2} \mathbf{Q}_{x y}^{\prime}+\sigma_{x z}^{2} \mathbf{Q}_{x z}^{\prime}+\sigma_{y z}^{2} \mathbf{Q}_{y z}^{\prime}$

(stochastic model 2)

where

$$
\begin{aligned}
& \mathbf{Q}_{z z}^{\prime}=\operatorname{diag}\left(\mathbf{Q}_{z z} \quad 0 \quad 000000\right) \\
& \mathbf{Q}_{x x}^{\prime}=\operatorname{diag}\left(\begin{array}{llllll}
0 & \mathbf{Q}_{x x} & 0 & 0 & 0 & 0
\end{array}\right)
\end{aligned}
$$

$$
\begin{aligned}
& \mathbf{Q}_{y y}^{\prime}=\operatorname{diag}\left(\begin{array}{llllll}
0 & 0 & \mathbf{Q}_{y y} & 0 & 0 & 0
\end{array}\right) \\
& \mathbf{Q}_{x y}^{\prime}=\operatorname{diag}\left(\begin{array}{llllll}
0 & 0 & 0 & \mathbf{Q}_{x y} & 0 & 0
\end{array}\right) \\
& \mathbf{Q}_{x z}^{\prime}=\operatorname{diag}\left(\begin{array}{lllllll}
0 & 0 & 0 & 0 & \mathbf{Q}_{x z} & 0
\end{array}\right) \\
& \mathbf{Q}_{y z}^{\prime}=\operatorname{diag}\left(\begin{array}{llllll}
0 & 0 & 0 & 0 & 0 & \mathbf{Q}_{y z}
\end{array}\right)
\end{aligned}
$$

is the same as that of Proposition 1.

Proof. The corollary follows as the only parameter depending on the stochastic model in Proposition 1 is $k_{j}^{n m}$, and the estimator presented in the proposition does not change by the stochastic model.

\subsection{The non-negative estimator of a DOVC}

Suppose that $\overline{\mathbf{w}}^{T} \mathbf{M} \overline{\mathbf{w}}$, where $\mathbf{M}$ is a symmetric matrix, is an unbiased estimator of $\mathbf{p}^{T} \boldsymbol{\sigma}$. The estimator is not necessarily positive, but if $\mathbf{M}$ is restricted to the class of matrices $\mathbf{M}=\mathbf{G G}^{T}$, where $\mathbf{G}$ is an arbitrary matrix of compatible dimensions, the quadratic form of $\overline{\mathbf{w}}^{T} \mathbf{M} \overline{\mathbf{w}}$ will be non-negative. A sufficient condition for the estimator to be unbiased in this case is (Sjöberg, 1984a)

$$
p_{i}=\operatorname{trace}\left\{\mathbf{G}^{T}\left(\mathbf{I}-\mathbf{A}^{0}\right) \mathbf{C}_{i}\left(\mathbf{I}-\mathbf{A}^{0}\right)^{T} \mathbf{G}\right\} \text {. }
$$

As pointed out by LaMotte (1973), unbiased, non-negative estimators satisfying this relation exist only in special cases. Here, the discussion is restricted to the unbiased estimation of individual VCs. In a first step, $\overline{\mathbf{w}}$ is transformed into

$$
\gamma_{i}=\mathbf{F}_{i} \overline{\mathbf{w}}
$$

where we have chosen the projection $\mathbf{F}_{i}$ as

$$
\mathbf{F}_{i}=\mathbf{I}-\mathbf{C}_{0 i} \mathbf{C}_{0 i}^{-} \text {and } \mathbf{C}_{0 i}=\mathbf{C}-\sigma_{i}^{2} \mathbf{C}_{i},
$$

with

$$
\mathbf{C}=\overline{\mathbf{B}} \mathbf{Q} \overline{\mathbf{B}}^{T} \text { and } \mathbf{C}_{i}=\overline{\mathbf{B}} \mathbf{Q}_{i} \overline{\mathbf{B}}^{T}
$$

Let us introduce $\mathbf{P}_{i}=\mathbf{F}_{i} \mathbf{C}_{i}\left(\mathbf{I}-\mathbf{A}^{0}\right)^{T} \mathbf{F}_{i}($ Sjöberg, 1984b) then

$$
\hat{\sigma}_{i}^{2}=\boldsymbol{\gamma}_{i}^{T} \mathbf{P}_{i}^{-} \boldsymbol{\gamma}_{i} / \operatorname{rank}\left(\mathbf{P}_{i}\right)
$$

where $\mathbf{P}_{i}^{-}$is any generalized inverse of $\mathbf{P}_{i}$, which is a BQUNE of the VC (see Sjöberg, 1984b; Eshagh and Sjöberg, 2008 for two ways of proof).

The MBQUNE was introduced by Eshagh and Sjöberg (2008) for cases where $\mathbf{F}_{i}=0$ and successfully implemented by Kiamehr and Eshagh (2008) to combine the geometric and gravimetric geoid models in Iran. It should be mentioned that $\mathbf{F}_{i}=0$ also occurs when the solutions of the GBVP are combined. The MBQUNE is defined when $\mathbf{F}_{i}=\mathbf{I}-\mathbf{Q}_{0 i} \mathbf{Q}_{0 i}^{-}$and $\mathbf{Q}_{0 i}=\mathbf{Q}-\sigma_{i}^{2} \mathbf{Q}_{i}$, as

$$
\boldsymbol{\gamma}_{i}=\mathbf{F}_{i} \mathbf{Q} \overline{\mathbf{B}}^{T} \mathbf{C}^{-} \overline{\mathbf{w}}=\mathbf{F}_{i} \hat{\boldsymbol{\varepsilon}}
$$

Based on normally distributed observations with zero expectation of errors, this holds also for $\boldsymbol{\gamma}_{i}$, yielding (Eshagh and Sjöberg, 2008):

$$
\tilde{\mathbf{P}}_{i}=\mathbf{F}_{i} \mathbf{Q} \overline{\mathbf{B}}^{T} \mathbf{C}^{-} \overline{\mathbf{B}} \mathbf{Q} \mathbf{F}_{i} .
$$

Proposition 2 The MBQUNE of a DOVC in the CAM based on stochastic model 1 for the vertical-vertical $(V V)$ solution 
is:

$$
\begin{aligned}
& \left(\hat{\sigma}_{\mathrm{VV}}^{2}\right)^{n m}=\left(\hat{\sigma}_{z z}^{2}\right)^{n m} \\
& =\frac{\left(c_{22}^{n m}-2 c_{12}^{n m}+c_{11}^{n m}\right)\left(\hat{\boldsymbol{\varepsilon}}_{z z}^{n m}\right)^{T}\left[\mathbf{Q}_{z z}\left(\mathbf{b}_{z z}^{n m}\right)^{T} \mathbf{b}_{z z}^{n m} \mathbf{Q}_{z z}\right]^{-} \hat{\boldsymbol{\varepsilon}}_{z z}^{n m}}{\left|\mathbf{C}^{n m}\right| \operatorname{rank}\left[\mathbf{Q}_{z z}\left(\mathbf{b}_{z z}^{n m}\right)^{T} \mathbf{b}_{z z}^{n m} \mathbf{Q}_{z z}\right]} \\
& \left|\mathbf{C}^{n m}\right| \neq 0
\end{aligned}
$$

Proof. Because of similar structures, the proof of this proposition will be presented with the proof of the next proposition.

Proposition 3 The MBQUNE of a DOVC based on stochastic model 1 for the horizontal-horizontal $(\mathrm{HH})$ and verticalhorizontal (VH) solution is:

$$
\begin{gathered}
\left(\hat{\sigma}_{i}^{2}\right)^{n m}=\frac{c_{k^{\prime} k^{\prime}}^{n m}\left(\hat{\boldsymbol{\varepsilon}}_{i}^{n m}\right)^{T}\left(\mathbf{P}_{i}^{n m}\right)^{-} \hat{\boldsymbol{\varepsilon}}_{i}^{n m}}{\left|\mathbf{C}^{n m}\right| \operatorname{rank}\left(\mathbf{P}_{i}^{n m}\right)} \\
\text { where } i=\left\{\begin{array}{ll}
\mathrm{HH} & k^{\prime}=2 \\
\mathrm{VH} & k^{\prime}=1
\end{array} \text { and }\left|\mathbf{C}^{n m}\right| \neq 0\right.
\end{gathered}
$$

where $\left(\hat{\boldsymbol{\varepsilon}}_{\mathrm{HH}}^{n m}\right)^{T}=\left[\begin{array}{lll}\hat{\boldsymbol{\varepsilon}}_{x x}^{n m} & \hat{\boldsymbol{\varepsilon}}_{y y}^{n m} & \hat{\boldsymbol{\varepsilon}}_{x y}^{n m}\end{array}\right]^{T},\left(\hat{\boldsymbol{\varepsilon}}_{\mathrm{VH}}^{n m}\right)^{T}=\left[\begin{array}{ll}\hat{\boldsymbol{\varepsilon}}_{x z}^{n m} & \hat{\boldsymbol{\varepsilon}}_{y z}^{n m}\end{array}\right]^{T}$,

$$
\begin{aligned}
\mathbf{P}_{\mathrm{HH}}^{n m}= & {\left[\begin{array}{lll}
\mathbf{Q}_{x x}\left(\mathbf{b}_{x x}^{n m}\right)^{T} \mathbf{b}_{x x}^{n m} \mathbf{Q}_{x x} & \mathbf{Q}_{x x}\left(\mathbf{b}_{x x}^{n m}\right)^{T} \mathbf{b}_{y y}^{n m} \mathbf{Q}_{y y} & \mathbf{Q}_{x x}\left(\mathbf{b}_{x x}^{n m}\right)^{T} \mathbf{b}_{x y}^{n m} \mathbf{Q}_{x y} \\
\mathbf{Q}_{y y}\left(\mathbf{b}_{y y}^{n m}\right)^{T} \mathbf{b}_{x x}^{n m} \mathbf{Q}_{x x} & \mathbf{Q}_{y y}\left(\mathbf{b}_{y y}^{n m}\right)^{T} \mathbf{b}_{y y}^{n m} \mathbf{Q}_{y y} & \mathbf{Q}_{y y}\left(\mathbf{b}_{y y}^{n m}\right)^{T} \mathbf{b}_{x y}^{n m} \mathbf{Q}_{x y} \\
\mathbf{Q}_{x y}\left(\mathbf{b}_{x y}^{n m}\right)^{T} \mathbf{b}_{x x}^{n m} \mathbf{Q}_{x x} & \mathbf{Q}_{x y}\left(\mathbf{b}_{x y}^{n m}\right)^{T} \mathbf{b}_{y y}^{n m} \mathbf{Q}_{y y} & \mathbf{Q}_{x y}\left(\mathbf{b}_{x y}^{n m}\right)^{T} \mathbf{b}_{x y}^{n m} \mathbf{Q}_{x y}
\end{array}\right] } \\
\mathbf{P}_{\mathrm{VH}}^{n m}= & {\left[\begin{array}{lll}
\mathbf{Q}_{x z}\left(\mathbf{b}_{x z}^{n m}\right)^{T} \mathbf{b}_{x z}^{n m} \mathbf{Q}_{x z} & \mathbf{Q}_{x z}\left(\mathbf{b}_{x z}^{n m}\right)^{T} \mathbf{b}_{y z}^{n m} \mathbf{Q}_{y z} \\
\mathbf{Q}_{y z}\left(\mathbf{b}_{y z}^{n m}\right)^{T} \mathbf{b}_{x z}^{n m} \mathbf{Q}_{x z} & \mathbf{Q}_{y z}\left(\mathbf{b}_{y z}^{n m}\right)^{T} \mathbf{b}_{y z}^{n m} \mathbf{Q}_{y z}
\end{array}\right] . }
\end{aligned}
$$

Proof. The adjustment model used in this derivation is the CAM. Therefore, by putting $\mathbf{A}=0$ in Eq. (13), we obtain

$$
\tilde{\mathbf{P}}_{i}=\mathbf{F}_{i} \mathbf{Q} \mathbf{B}^{T} \mathbf{C}^{-1} \mathbf{B} \mathbf{Q} \mathbf{F}_{i},
$$

where $i=\mathrm{VV}, \mathrm{HH}$ and $\mathrm{VH}$, respectively, and, according to stochastic model 1 , we obtain

$$
\begin{aligned}
& \mathbf{F}_{\mathrm{VV}}=\operatorname{diag}\left(\left[\begin{array}{llllll}
I & 0 & 0 & 0 & 0 & 0
\end{array}\right]\right), \\
& \mathbf{F}_{\mathrm{HH}}=\operatorname{diag}\left(\left[\begin{array}{llllll}
0 & I & I & I & 0 & 0
\end{array}\right]\right), \\
& \mathbf{F}_{\mathrm{VH}}=\operatorname{diag}\left(\left[\begin{array}{llllll}
0 & 0 & 0 & 0 & I & I
\end{array}\right]\right) .
\end{aligned}
$$

Finally, by inserting Eqs. (32a)-(32c) into Eq. (31), we have

$$
\mathbf{P}_{\mathrm{VV}}^{n m}=\left(c_{12}^{n m}-2 c_{12}^{n m}+c_{12}^{n m}\right)\left|\mathbf{C}^{n m}\right|^{-1} \mathbf{Q}_{z z}\left(\mathbf{b}_{z z}^{n m}\right)^{T} \mathbf{b}_{z z}^{n m} \mathbf{Q}_{z z},
$$

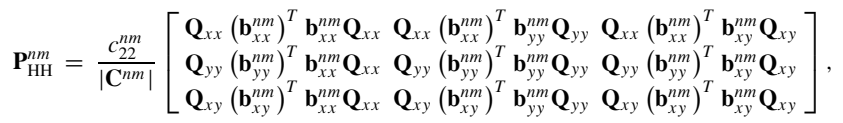

$\mathbf{P}_{\mathrm{VH}}^{n m}=\frac{c_{11}^{n m}}{\left|\mathbf{C}^{n m}\right|}\left[\begin{array}{l}\mathbf{Q}_{x z}\left(\mathbf{b}_{x z}^{n m}\right)^{T} \mathbf{b}_{x z}^{n m} \mathbf{Q}_{x z} \mathbf{Q}_{x z}\left(\mathbf{b}_{x}^{n m}\right)^{T} \mathbf{b}_{y z}^{n m} \mathbf{Q}_{y z} \\ \mathbf{Q}_{y z}\left(\mathbf{b}_{y z}^{n m}\right)^{T} \mathbf{b}_{x z}^{n m} \mathbf{Q}_{x z} \mathbf{Q}_{y z}\left(\mathbf{b}_{y z}^{n m}\right)^{T} \mathbf{b}_{y z}^{n m} \mathbf{Q}_{y z}\end{array}\right]$.

Propositions 2 and 3 follow by inserting Eqs. (33a)-(33c) into Eq. (28) and then considering Eq. (29).

Proposition 4 The MBQUNE of a DOVC based on stochastic model 2 is

$$
\left(\hat{\sigma}_{i}^{2}\right)^{n m}=\frac{\left[\mathbf{b}_{i}^{n m} \mathbf{Q}_{i}\left(\mathbf{b}_{i}^{n m}\right)^{T}\right] c_{k k}^{n m}\left(\hat{\boldsymbol{\varepsilon}}_{i}^{n m}\right)^{T}\left[\mathbf{Q}_{i}\left(\mathbf{b}_{i}^{n m}\right)^{T} \mathbf{b}_{i}^{n m} \mathbf{Q}_{i}\right]^{-} \hat{\boldsymbol{\varepsilon}}_{i}^{n m}}{\left|\mathbf{C}^{n m}\right| \operatorname{rank}\left[\mathbf{Q}_{i}\left(\mathbf{b}_{i}^{n m}\right)^{T} \mathbf{b}_{i}^{n m} \mathbf{Q}_{i}\right]},
$$$$
\left|\mathbf{C}^{n m}\right| \neq 0,
$$

where

$$
i=\left\{\begin{array}{ll}
x x, y y, x y & k=2 \\
x z, y z & k=1
\end{array} .\right.
$$

Proof. The mathematical proof of the proposition is very similar to the proof provided for Proposition 3. The differences are just due to projector $\mathbf{F}_{i}, i=x x, y y, x y, x z$ and $y z$ and stochastic model 2, where

$$
\begin{aligned}
& \mathbf{F}_{x x}=\operatorname{diag}\left(\left[\begin{array}{llllll}
0 & I & 0 & 0 & 0 & 0
\end{array}\right]\right), \\
& \mathbf{F}_{y y}=\operatorname{diag}\left(\left[\begin{array}{llllll}
0 & 0 & I & 0 & 0 & 0
\end{array}\right]\right), \\
& \mathbf{F}_{x y}=\operatorname{diag}\left(\left[\begin{array}{lllllll}
0 & 0 & 0 & I & 0 & 0
\end{array}\right]\right), \\
& \mathbf{F}_{x z}=\operatorname{diag}\left(\left[\begin{array}{llllll}
0 & 0 & 0 & 0 & I & 0
\end{array}\right]\right), \\
& \mathbf{F}_{y z}=\operatorname{diag}\left(\left[\begin{array}{llllll}
0 & 0 & 0 & 0 & 0 & I
\end{array}\right]\right),
\end{aligned}
$$

and

$$
\begin{aligned}
& \mathbf{C}_{i}^{n m}=\mathbf{b}_{i}^{n m} \mathbf{Q}_{i}\left(\mathbf{b}_{i}^{n m}\right)^{T}\left[\begin{array}{ll}
1 & 0 \\
0 & 0
\end{array}\right], i=x x, y y \text { and } x y \\
& \mathbf{C}_{j}^{n m}=\mathbf{b}_{j}^{n m} \mathbf{Q}_{j}\left(\mathbf{b}_{j}^{n m}\right)^{T}\left[\begin{array}{ll}
0 & 0 \\
0 & 1
\end{array}\right], j=x z \text { and } y z .
\end{aligned}
$$

The proposition is proved by substituting Eqs. (35a)-(35b) and Eqs. (34a)-(34e) into Eq. (28) and then considering Eq. (29) and some further simplifications.

\subsection{Numerical investigation on the combination using the DOVC estimation}

As the combined solution using the DOVC estimation process has now been tested, this process can be considered in two different cases: (1) stochastic model 1 in which one DOVC is estimated for each integral solution, denoted combined solution 1; (2) stochastic model 2 in which one DOVC is estimated for each type of observation (six DOVCs), denoted combined solution 2. This discussion begins with combined solution 1. Figure 3 shows the true and simple mean solution with respect to this solution.

In this figure, it can be seen that the combined solution 1 is very close to the simple mean solution. In some degrees it is superior and in other degrees it is inferior with respect

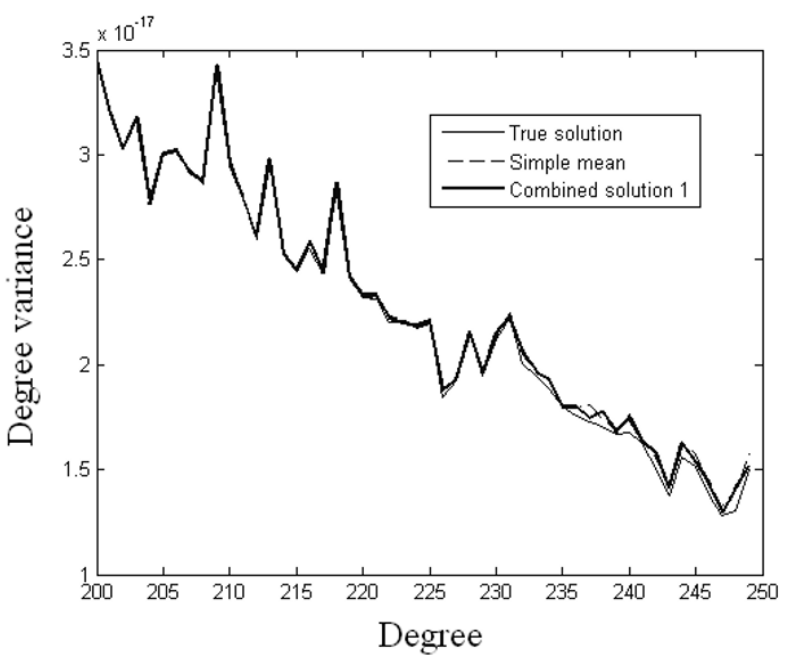

Fig. 3. True solution, simple mean and combined solution 1. 
to the simple mean. In general, we cannot say that the combined solution 1 is better than the simple mean unless we consider their differences. Such differences are presented in Fig. 7.

The DOVC differs by each degree and order. This means that for some degrees and orders, the observables can have different weights in the combination. Another important matter is that the negative DOVC can only be seen for zonal terms. The non-negative $\mathrm{VC}$ estimators can be used to solve this problem. Here, the MBQUNE is used to estimate the DOVC, but there is a practical problem to using this estimator. First, there are the matrix products $\mathbf{Q}_{z z}\left(\mathbf{b}_{z z}^{n m}\right)^{T} \mathbf{b}_{z z}^{n m} \mathbf{Q}_{z z}$ in Proposition 3. $\mathbf{b}_{z z}^{n m}$ is a row vector and its dimensions depend directly on the resolution of the data used. In our case, a $30^{\prime} \times 30^{\prime}$ resolution has been considered for the SGG data. Therefore, this vector will have $360 \times 720=259,200$ column-wise elements. Consequently, $\left(\mathbf{b}_{z z}^{n m}\right)^{T} \mathbf{b}_{z z}^{n m}$ will be $259,200 \times 259,200$. As Proposition 2 shows, $\mathbf{Q}_{z z}\left(\mathbf{b}_{z z}^{n m}\right)^{T} \mathbf{b}_{z z}^{n m} \mathbf{Q}_{z z}$ should be inverted by a generalized inverse (Bjerhammar, 1973). This action represents a very complicated manipulation for ordinary computers, and the situation becomes even worse when estimating the DOVCs of the $\mathrm{VH}$ and $\mathrm{HH}$ solutions as the dimension of the matrices to be inverted is even larger. Consequently, we can say that the MBQUNE, which was theoretically investigated, is, at the present time, not practical. Hopefully, with the continued development of supercomputers and computer technology, this problem with be solvable. It will therefore be left for future studies.

In order to escape from the negative DOVCs, let us consider the simple mean for the combination. This investigation shows larger differences for lower orders than higher ones in the three integral solutions of the GBVP, and after order 10, the geopotential coefficients, which are being computed by the integral solutions, are very close or more or less the same. It should be considered that the magnitude of the low-order harmonic coefficients is larger than that of the higher order ones. Therefore, for high orders, which are approximately the same, it is not important whether either the simple mean or combined method by DOVC is used.

In order to present the performance of the DOVC estimation process, let us consider $n=200$ and $m=2$ coefficients for which the negative DOVC does not happen.

Figure 4 presents the speed of convergence of the DOVC ratios to 1 . The ratio converges quickly to 1 in the $\mathrm{VV}$ solution, but the convergence is very slow for the $\mathrm{HH}$ and VH solutions. Here, the DOVC ratios have been presented up to 50 iterations. Based on the results of the present investigation, the DOVC ratios of these solutions do not converge to 1 even up to 500 iterations, but they do come closer to 1 . In order to provide some idea ont the DOVC values, these are presented in Table 1. This table shows the DOVC of the VV, $\mathrm{HH}$ and $\mathrm{VH}$ solutions; it should be noted that the DOVC estimation process is performed for the cosine and sine coefficients separately. The first impression is of larger values for the DOVCs of $\hat{S}_{200,2}$ than of $\hat{C}_{200,2}$. However, the relative weight is important in our re-weighing process. The DOVC estimation tries to balance between the accuracy of the a priori variance factor and misclosure vector. Since the mis-closures are very small, the
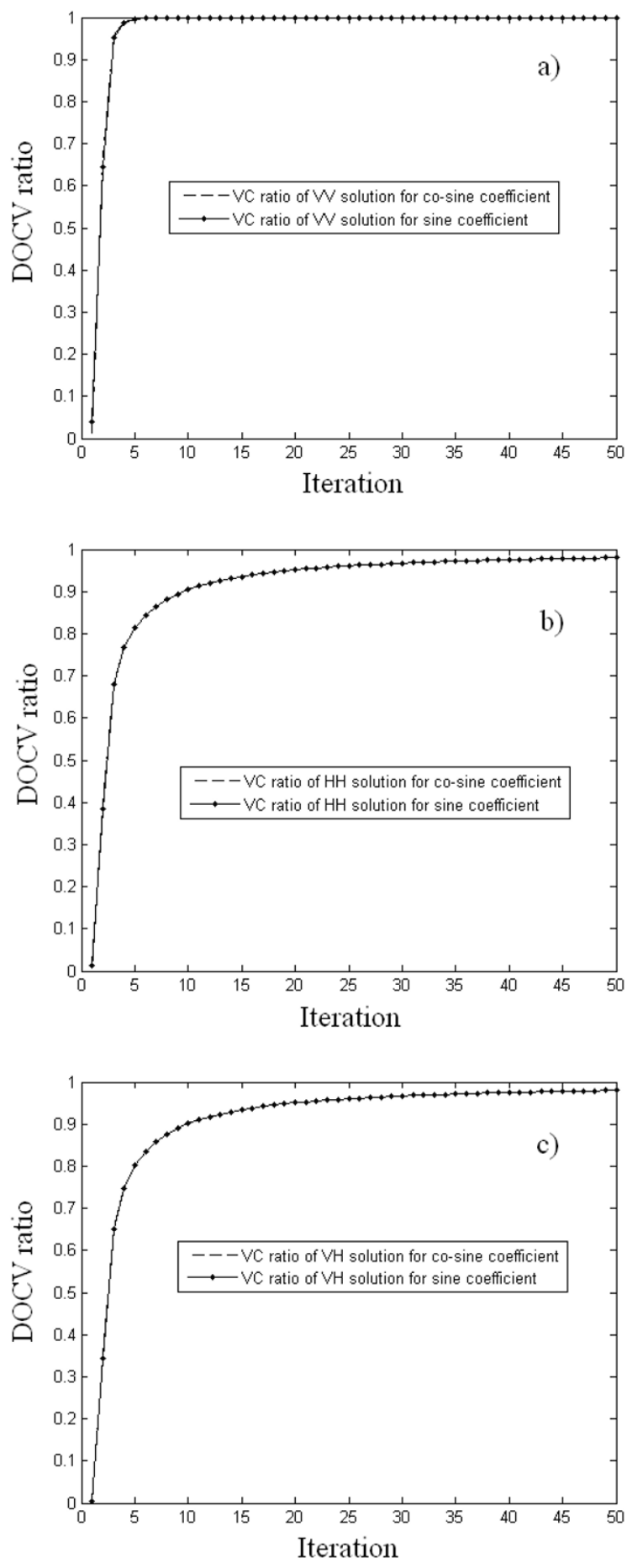

Fig. 4. DOVC ratios for (a) VV solution, (b) $\mathrm{HH}$ solution and (c) $\mathrm{VH}$ solution for $n=200$ and $m=2$.

DOVC also comes out small. Another objective is to obtain the smallest DOVC for the VH solution. However, it can be seen that the DOVC for the HH solution is smaller than that for the VV solution. If Proposition 1 is considered, we can see that the only parameter which is dependent on the stochastic model is $k_{j}$, a parameter presented in Eqs. (21a)(21c). The explanation of why a smaller DOVC is obtained for the $\mathrm{HH}$ solution is that $k_{\mathrm{HH}}$ becomes larger than $k_{\mathrm{VV}}$ as a result of including three quadratic forms (see Eq. (21b)). 
Table 1. DOVCs of the VV, HH and VH solution for $n=200$ and $m=2$.

\begin{tabular}{ccc}
\hline & $\hat{C}_{200,2}$ & $\hat{S}_{200,2}$ \\
\hline$\left(\hat{\sigma}_{\mathrm{VV}}^{2}\right)^{200,2}$ & $7.97 \times 10^{-9}$ & $2.41 \times 10^{-6}$ \\
$\left(\hat{\sigma}_{\mathrm{HH}}^{2}\right)^{200,2}$ & $7.75 \times 10^{-11}$ & $2.34 \times 10^{-8}$ \\
$\left(\hat{\sigma}_{\mathrm{VH}}^{2}\right)^{200,2}$ & $2.27 \times 10^{-11}$ & $6.87 \times 10^{-9}$ \\
\hline
\end{tabular}

Table 2. DOVC ratios for VV, HH and VH solutions based on stochastic model 1 for $n=200, m=2, n=230, m=5, n=245, m=8$.

\begin{tabular}{cccc}
\hline & $n=200$ & $n=230$ & $n=245$ \\
& $m=2$ & $m=5$ & $m=8$ \\
\hline$\left(\hat{\sigma}_{\mathrm{VH}}^{2}\right)^{n m} /\left(\hat{\sigma}_{\mathrm{HH}}^{2}\right)^{n m}$ & 0.29 & 0.08 & 0.04 \\
$\left(\hat{\sigma}_{\mathrm{VV}}^{2}\right)^{n m} /\left(\hat{\sigma}_{\mathrm{HH}}^{2}\right)^{n m}$ & 408.32 & 404.79 & 401.04 \\
\hline
\end{tabular}

Let us consider the ratio of the DOVCs in these solutions to show that the relative weights in the solutions of both cosine and sine coefficients are approximately the same.

According to Table 2 we should not bother with the differences in magnitude as their relative weights are the same. The difference is due to the magnitude of the mis-closure vector for the optimal spectral combination of cosine and sine coefficients.

Now let us return to stochastic model 2, which was presented in Corollary 1. Again, in this case the MBQUNE is not a practical way to obtain the non-negative estimates for the DOVCs. Therefore, we confine ourselves just to the cases where the positive DOVC emerges. Each time a negative DOVC is derived, the simple mean is used for the combination. Negative DOVC was also seen for zonal terms and for some other terms. According to the investigation reported here, negative DOVCs frequently occur in the combined solution 2. However, they mostly belong to the orders higher than 14 and, as already mentioned, no significant differences among the $\mathrm{VV}, \mathrm{HH}$ and $\mathrm{VH}$ solutions for the higher orders were observed in this study. The result of this method of the spectral combination is presented in Fig. 6.

Figure 5 clearly shows that the combined solution 2 is very close to the true solution so that one curve is seen in the figure. For better visualization of these solutions we consider the differences between these solutions and the true solution in Fig. 6. In Fig. 6, the dash line ( - - ) stands for the difference between the true and the simple mean solutions, the solid line $(-)$ is the difference between the combined solution 1 and the true one and the thick solid line $(-)$ is the difference between the combined solution 1 and the true one. Figure 5 shows that the even degrees are well estimated in the combined solution 2, but that the estimates for the odd degrees are the same as the simple mean. Therefore, the combined solution 1 is slightly superior to the simple mean. However, the combined solution 2 is superior to the other solutions and very much closer to the true solution. Since the VH solution was closer to the true solution than the VV and $\mathrm{HH}$ solutions, the difference between the true and the $\mathrm{VH}$ solutions in Fig. 6 was plotted in order to demonstrate that the combined solution 1 is a solution between the simple mean and the VH solution; however, as can be seen, the

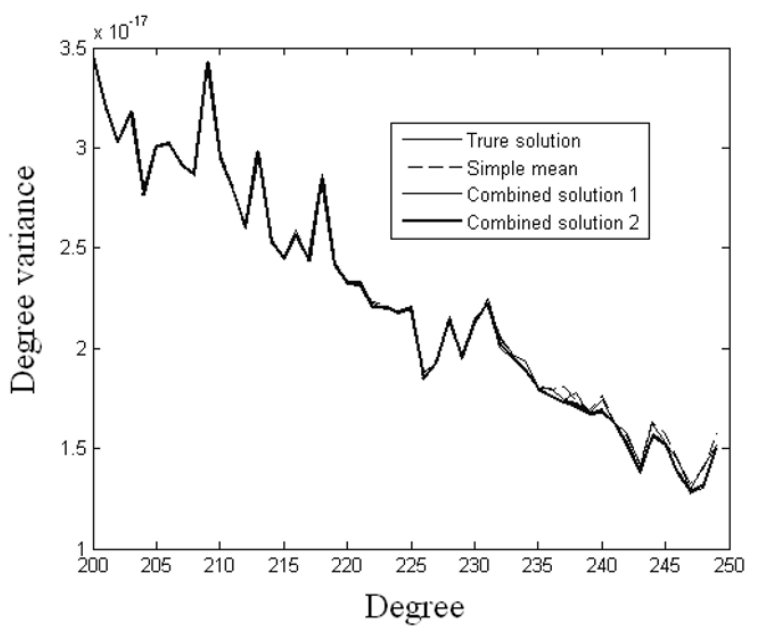

Fig. 5. Signal degree variances of the true, simple mean and combined solutions 1 and 2 .

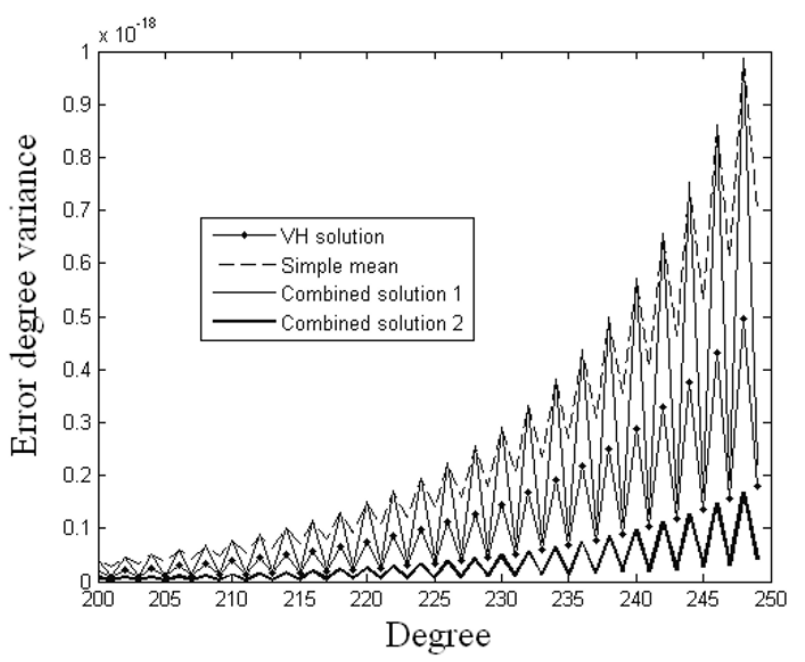

Fig. 6. Error degree variances of the true and VH solution and simple solution and combined solutions 1 and 2.

combined solution 2 is even superior to the $\mathrm{VH}$ solution. According to the numerical investigations, the DOVC converges very quickly in this combined solution. After about three iterations, all of the DOVC ratios converge to 1 . This may be due to the stochastic model that we have used in which all observations were considered to be independent. The major problem of this combination method is that negative DOVCs are frequently obtained for the orders above 14 due to the fact that the three solutions provide approximately the same result after this order of magnitude. However, everywhere they differ, the combined solution is used; therefore, selection of the simple mean is quite reasonable for those orders for which the DOVCs come out negative.

Figure 7 shows that the DOVC ratios for $n=200$ and $m=2$ of $T_{z z}, T_{x x}, T_{y y}$ and $T_{x z}$ are very similar and that they converge to 1 in three iterations. This is not like the combined solution 1 in which the DOVC ratios have uniform convergence. In the combined solution 2, the DOVC ratios oscillate before convergence. The DOVC ratios of $T_{x y}$ and $T_{y z}$ in the combined solution 2 are very similar as 

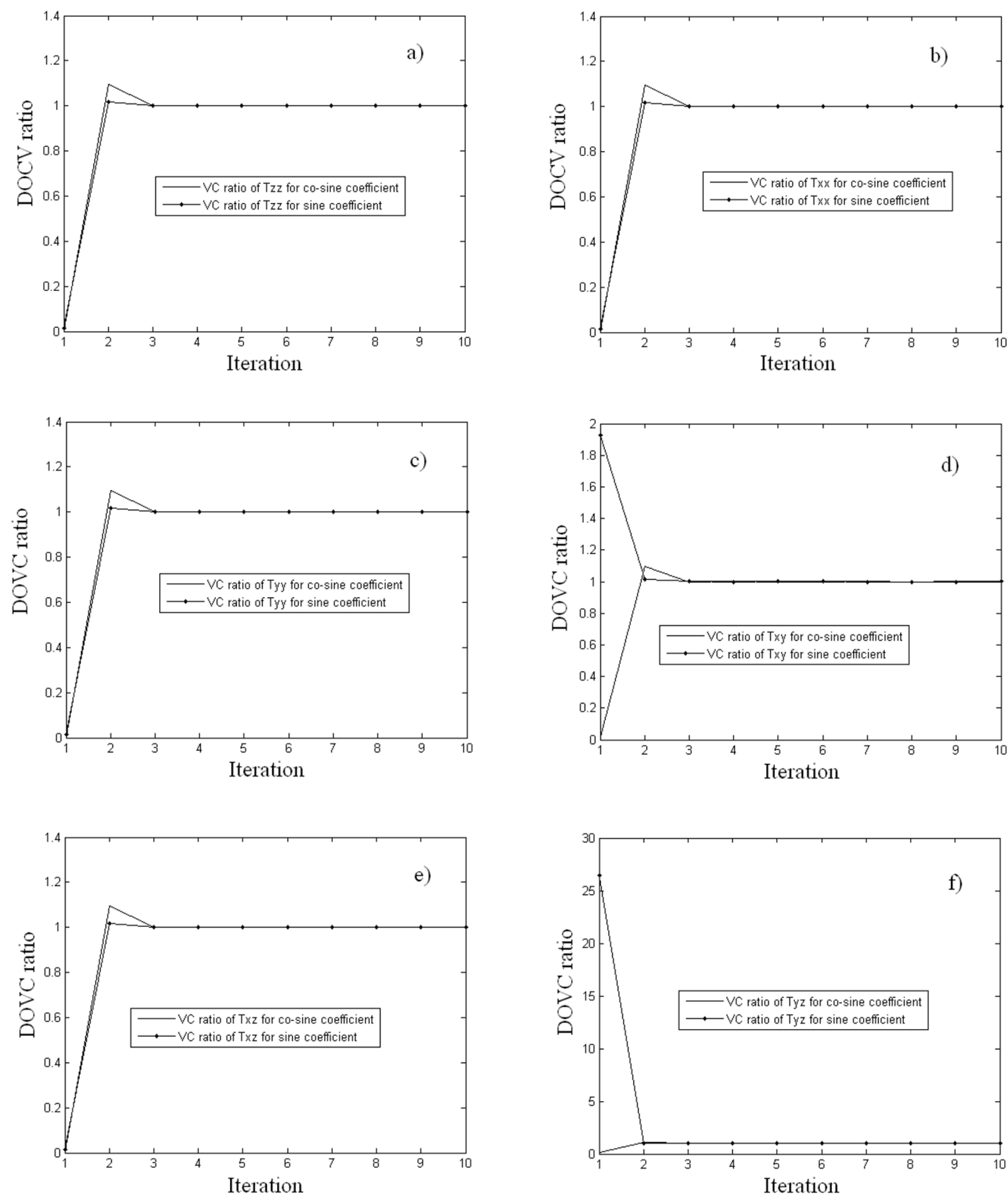

Fig. 7. DOVC ratios for (a) $T_{z z}$, (b) $T_{x x}$, (c) $T_{y y}$, (d) $T_{x y}$, (e) $T_{x z}$ and (f) $T_{y z}$.

they are decreasing for the cosine coefficients and increasing for sine coefficients.

Let us tabulate the DOVCs of these observations in the combined solution 2 in Table 3. Table 3 illustrates the DOVCs of $T_{z z}, T_{x x}, T_{y y}, T_{x y}, T_{x z}$ and $T_{y z}$, respectively, for $n=200$ and $m=2$ as this table shows $\left(\hat{\sigma}_{x x}^{2}\right)^{200,2}=$ $\left(\hat{\sigma}_{y y}^{2}\right)^{200,2}$ in both sine and cosine coefficients. The reason for this is that the coefficients matrices of $T_{x x}$ and $T_{y y}$ are exactly the same in magnitude, differing only in sign.
Consequently, the errors which are propagated based on both coefficients will be the same, which is why the same DOVCs are obtained for these types of observations. According to the Laplace equation, the DOVC of $T_{z z}$ should be in order of the DOVCs of $T_{y y}$ and $T_{x y}$, which is why was obtained $\left(\hat{\sigma}_{z z}^{2}\right)^{200,2}$ in the order of $\left(\hat{\sigma}_{x x}^{2}\right)^{200,2}$ and $\left(\hat{\sigma}_{y y}^{2}\right)^{200,2}$. The other DOVC of $T_{x z}$ is also in order of $\left(\hat{\sigma}_{z z}^{2}\right)^{200,2},\left(\hat{\sigma}_{x x}^{2}\right)^{200,2}$ and $\left(\hat{\sigma}_{y y}^{2}\right)^{200,2}$; it is also closer to $\left(\hat{\sigma}_{z z}^{2}\right)^{200,2}$, and it seems that there is more or less the same power of the gravita- 
Table 3. DOVCs of $T_{z z}, T_{x x}, T_{y y}, T_{x y}, T_{x z}$ and $T_{y z}$ for $n=200$ and $m=2$.

\begin{tabular}{ccc}
\hline & $\hat{C}_{200,2}$ & $\hat{S}_{200,2}$ \\
\hline$\left(\hat{\sigma}_{z z}^{2}\right)^{200,2}$ & $5.74 \times 10^{-9}$ & $1.6 \times 10^{-6}$ \\
$\left(\hat{\sigma}_{x x}^{2}\right)^{200,2}$ & $5.64 \times 10^{-9}$ & $1.57 \times 10^{-6}$ \\
$\left(\hat{\sigma}_{y y}^{2}\right)^{200,2}$ & $5.64 \times 10^{-9}$ & $1.57 \times 10^{-6}$ \\
$\left(\hat{\sigma}_{x y}^{2}\right)^{200,2}$ & $7.00 \times 10^{-7}$ & $1.96 \times 10^{-4}$ \\
$\left(\hat{\sigma}_{x z}^{2}\right)^{200,2}$ & $5.72 \times 10^{-9}$ & $1.60 \times 10^{-6}$ \\
$\left(\hat{\sigma}_{y z}^{2}\right)^{200,2}$ & $9.60 \times 10^{-6}$ & $2.7 \times 10^{-4}$ \\
\hline
\end{tabular}

Table 4. DOVCs ratios of $T_{z z}, T_{x x}, T_{y y}, T_{x y}, T_{x z}$ and $T_{y z}$ based on stochastic model 1 for $n=200, m=2, n=230, m=5, n=245$, $m=8$.

\begin{tabular}{crcc}
\hline & $n=200$ & $n=230$ & $n=245$ \\
& $m=2$ & $m=5$ & $m=8$ \\
\hline$\left(\hat{\sigma}_{x x}^{2}\right)^{n m} /\left(\hat{\sigma}_{z z}^{2}\right)^{n m}$ & 0.98 & 0.99 & 1.00 \\
$\left(\hat{\sigma}_{y y}^{2}\right)^{n m} /\left(\hat{\sigma}_{z z}^{2}\right)^{n m}$ & 0.98 & 0.99 & 1.00 \\
$\left(\hat{\sigma}_{x y}^{2}\right)^{n m} /\left(\hat{\sigma}_{z z}^{2}\right)^{n m}$ & 122.00 & 30.92 & 15.24 \\
$\left(\hat{\sigma}_{x z}^{2}\right)^{n m} /\left(\hat{\sigma}_{z z}^{2}\right)^{n m}$ & 0.99 & 1.00 & 1.00 \\
$\left(\hat{\sigma}_{y z}^{2}\right)^{n m} /\left(\hat{\sigma}_{z z}^{2}\right)^{n m}$ & 1671.50 & 406.13 & 195.41 \\
\hline
\end{tabular}

tional signal in $T_{z z}$ and $T_{x z}$. Larger DOVCs are related to $T_{x y}$ and $T_{y z}$, as presented in Table 3. As already explained, the important matter in re-weighting least-squares process is not the magnitude of the DOVC but the weights of the observations relative to each other (see Table 4).

Details of the re-weighting process are shown in this example using the DOVC estimation procedure. However, according to the numerical investigations reported here, the general pattern of these DOVCs more or less follows the same procedure in other degrees and orders. Table 4 suggests that $T_{x y}$ and $T_{y z}$ contribute relatively more in the combination of higher degrees and orders. Also, $T_{x y}$ seems to contain more power than $T_{y z}$, even in higher degrees and orders in the combination based on stochastic model 2 .

The numerical studies show the capability of the DOVCs to improve the observation weights. Here, we assumed that the observations are contaminated with white noise and that they are independent. The white noise can be an approximation for the coloured noise if the observations are not highly correlated; otherwise, the presented method is not suited for such applications. Here, we have considered GBVPs, but if we want to combine solutions of different geodetic boundary value problems, the presented method will be relevant. The other assumption is to have a global coverage of data, which is a necessary condition for solving the GBVP and determining the gravity field using integral formulas; this is not a restriction for the combination method using DOVC presented here.

\section{Conclusions and Recommendations}

In the study reported here, the VCE concept was not used in the standard way. The main goal of this new approach is not to estimate the VCs for the observable factors, but to find the observation weights for these and to absorb the discretization error of the integral solutions. According to our numerical studies, in the case where one DOVC is considered for each type of observation, the spectral combination is easier to use and the DOVC ratios converge to 1 faster than in the cases where DOVCs were estimated for each solution. The examples presented here show that the relative weight of the observations will be more or less the same for both sine and cosine geopotential coefficients and the magnitude of the DOVCs do not play important role as they can differ by degree and order. The negative VC occurred for the zonal terms and the degrees above 6 and 14 in combined solutions 1 and 2, respectively, but since the aim of this study is to combine the spectral solutions of GBVP, the simple mean was used for the combination. The MBQUNE of the VCs is not practical for the time being as it require manipulations with matrices with very large dimensions.

It is suggested that the equation of the CAMs be combined in the simplest possible way. The formulas presented in this study are well-suited for the GBVP, but for combining other solutions of the boundary value problem, it will be necessary to reconstruct the mathematical models and simplify them in order to obtain some simple formulas for the DOVC estimation; otherwise, it will be necessary to work with the matrices with large dimensions. The method presented here suffers from the resolution of the SGG data as the size of the functional vector increases. Also for each degree and order, the VCE process is iterated and postponed the solution.

Acknowledgments. The author would like to express his gratitude to his supervisor Prof. L. E. Sjöberg for his kind and great assistance during this study and also for technical editing of this paper. The Swedish National Space Board is acknowledged for the financial support project no. 63/07:1. The author would like to express his appreciation to the unknown reviewers for their constructive remarks. Professor Takeshi Sagiya is thanked for his editorial role in improving the quality of this paper.

\section{References}

Albertella, A., F. Sacerdote, and F. Sansò, Geodetic calculus with blockaverages observations on the sphere, Surv. Geophys., 14(4-5), 395-402, 1993.

Bjerhammar, A., Theory of Errors and Generalized Matrix Inverses, 420 pp., Elsevier Scientific Publ Co., Amsterdam, 1973.

Bjerhammar, A., A stochastic approach to the mixed boundary value problem in physical geodesy, in Geodesy in Transition, A volume dedicated to Helmut Moritz on the occasion of his 50th birthday, edited by Schwarz, K. P. and Lachapelle, G., Division of Surveying engineering, p. 25-42, The University of Calgary, Canada, 1983.

Eshagh, M., On Satellite Gravity Gradiometry, 222 pp., Doctoral thesis in Geodesy, Royal Institute of Technology, Stockholm, Sweden, 2009.

Eshagh, M. and L. E. Sjöberg, The modified best quadratic unbiased nonnegative estimator (MBQUNE) of variance components, Stud. Geophys. Geod., 52, 305-320, 2008.

Gelderen, M. and R. Rummel, The solution of the general boundary value problem by least-squares, J. Geod., 75(1), 1-11, 2001.

Gelderen, M. and R. Rummel, Corrections to "The solution of the general geodetic boundary value problem by least squares", J. Geod., 76(2), 121-122, 2002.

Grafarend, E., Linear and Non-linear Models, Fixed Effects, Random Effects, and Mixed Models, 752 pp., Springer Verlag, 2006.

Kiamehr, R. and M. Eshagh, Estimating variance components of ellipsoidal, orthometrc and geoidal heights through the GPS/leveling network in Iran, J. Earth Space Phys., 34(3), 1-13, 2008.

Koop, R., Global gravity field modeling using satellite gravity gradiometry, Publ Geodesy, New series, No. 38., 240 pp., Netherland Geodetic Commission, Delft, 1993.

Kusche, J., A Monte-Carlo technique for weight estimation in satellite 
geodesy, J. Geod., 76(6-7), 641-652, 2003.

LaMotte, L. R., On non-negative Quadratic unbiased estimation of variance components, J. Am. Stat. Assoc., 68(343), 728-730, 1973.

Lemoine, F. G., S. C. Kenyon, J. K. Factor, R. G. Trimmer, N. K. Pavlis,

D. Chinn, C. M. Cox, S. M. Klosko, S. B. Luthcke, M. H. Torrence, Y. M. Wang, R. G. Williamson, E. C. Pavlis, R. H. Rapp, and T. R. Olson, Geopotential model EGM96, NASA/TP-1998-206861, Goddard Space Flight Center, Greenbelt, 1998.

Martinec, Z., Green's function solution to spherical gradiometric boundary-value problems, J. Geod., 77, 41-49, 2003.

Rao, C. R., Estimation of variance components-MINQUE theory, J. Multivariate Anal., 1, 257-275, 1971.

Rao, C. R. and J. Kleffe, Estimation of Variance Components and Applications, 370 pp., North-Holland, Amsterdam, 1988.

Rummel, R., Spherical spectral properties of the Earth's gravitational potential and its first and second derivatives, Geodetic boundary value problems in view of the one centimetre geoid, in Lecture notes in Earth sciences, edited by Sanso, F. and Rummel, R., 359-401, 1997.

Rummel, R., F. Sanso, M. Gelderen, R. Koop, E. Schrama, M. Brovelli, F. Migiliaccio, and F. Sacerdote, Spherical harmonic analysis of satellite gradiometry, Publ Geodesy, New Series, No. 39, 124 pp., Netherlands Geodetic Commission, Delft, 1993.

Sanso, F. and G. Sona, The theory of optimal linear estimation for continue fields of measurements, Manus. Geod., 20(3), 204-230, 1995.

Sharifi, M. A., Satellite to Satellite Tracking in the Space-wise Approach, 162 pp., PhD dissertation, Geoätisches Institute der Universität Sttut- gart, Germany, 2006.

Sjöberg, L. E., Least-squares combination of satellite harmonics and integral formulas in physical geodesy, Gerlands Beitr. Geophys., Leipzig, 89(5), 371-377, 1980.

Sjöberg, L. E., Least-squares combination of terrestrial and satellite data in physical geodesy, Ann. Geophys., t. 37, fasc, 25-30, 1981.

Sjöberg, L. E., Unbiased estimation of variance-components in condition adjustment with unknowns-a MINQUE approach, ZfV, 108, 9, 1983.

Sjöberg, L. E., Least-Squares modification of Stokes' and Vening-Meinez' formula by accounting for truncation and potential coefficients errors, Manusc. Geod., 9, 209-229, 1984a.

Sjöberg, L. E., Non-negative variance component estimation in the GaussHelmert Adjustment model, Manus. Geod., 9, 247-280, 1984b.

Sneeuw, N., A Semi-analytical Approach to Gravity Field Analysis From Satellite Observations, 120 pp., Deutsche Geodaetische Kommission, Reihe C, Hlft, Nr. 527, 2000.

Wenzel, H. G., Zur Geoidbestimmung durch kombination von schwereanomalien und einem kugelfuncationsmodell mit hilfe von integralformeln, ZfV, 106(3), 102-111, 1981.

$\mathrm{Xu}, \mathrm{P}$., Y. Shen, Y. Fukuda, and Y. Liu, Variance components estimation in linear inverse ill-posed models, J. Geod., 80(2), 69-81, 2006.

Xu, P., Y. Liu, Y. Shen, and Y. Fukuda, Estimability analysis of variance and covariance components, J. Geod., 81(9), 593-602, 2007.

M. Eshagh (e-mail: eshagh@kth.se) 